\title{
Static Threshold Pressure Gradient of Micro-Channels
}

\author{
F. Q. Song ${ }^{1, *}$
}

${ }^{1}$ Department of Physics, Zhejiang Normal University, Jinhua, Zhejiang 321004, China

Email: songfuquan@tom.com

\begin{abstract}
The exploitation of oil fields and laboratory experiments exhibit the existence of Threshold Pressure Gradient(TPG) of liquid flow in low permeability porous media. That is to say, only the actual pressure gradient imposed is larger than TPG, the liquid can flow in porous media. At the same time, as the developing of Micro Electro Mechanism System(MEMS), some micro size effects are showed by some researchers, but the conclusions are not always accordant with each other. In this paper, an experiment setup is devised to measure the TPG of micro-channels by static water column method, and the TPG of water is measured in silicon micro-channels with diameter ranging from $50 \sim 320 \mu \mathrm{m}$. The results of experiments show the existence of TPG in micro-channel, which increases with the hydrodynamic diameter decreasing. The relation between TPG and diameter is according to single$\log$ law. Finally, The mechanism of micro size effects is discussed by facial force between liquid and solid and theory of boundary liquid, but the explanation is still not complete, and needs further study for scale smaller than micron and in flow states.
\end{abstract}

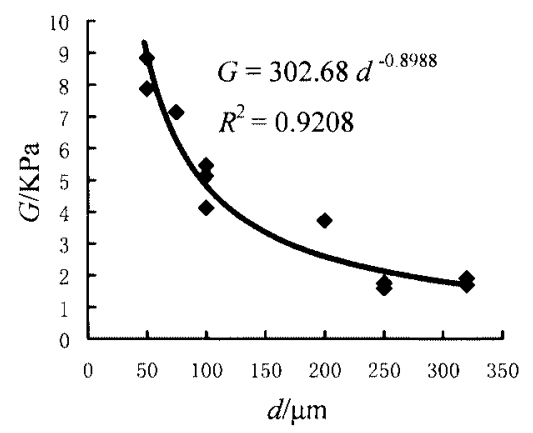

Figure: The TPG of micro-channels

\section{REFERENCES}

1. Gelbunuv A.T. Oil development of abnormal reservoirs. Petroleum Industry of China (in Chinese), 1987: 181

2. Huang Y.Z. The mechanism of flow in low permeability reservoirs. Petroleum Industry of China, (in Chinese), 1998

3. Ho C.M., Tai Y. C. Micro-Electro-Mechanical-Systems (MEMS) and fluid flows. Annu. Rev. Fluid Mech., 1998; 30: 579-612 